\title{
Sensitivity to Prior Specification in Bayesian Identification of Autoregressive Time Series Models
}

\author{
Ayman A Amin \\ Department of Statistics, Mathematics and Insurance \\ Faculty of Commerce, Menoufia University, Egypt \\ ayman.aamin@gmail.com
}

\begin{abstract}
In this paper we use the Kullback-Leibler divergence to measure the distance between the posteriors of autoregressive (AR) model order, aiming to evaluate mathematically the sensitivity of the model identification to different types of priors of the model parameters. In particular, we consider three priors for the AR model coefficients, namely Jeffreys', g, and natural conjugate priors, and three priors for the model order including uniform, arithmetic, and geometric priors. Using a large number of Monte Carlo simulations with various values of the model coefficients, model order, and sample size, we evaluate the impact of the posteriors distance in the accuracy of the model identification. Simulation study results show that the posterior of the model order is sensitive to prior distributions, and the highest accuracy of the model identification is obtained from the posterior resulting from the g-prior. Same results are obtained from the application to real-world time series datasets.
\end{abstract}

Keywords: Distance of posteriors, Kullback-Leibler divergence, Jeffreys' prior, g-prior, Natural conjugate prior.

\section{Introduction}

Prior specification plays an important role in the Bayesian analysis of time series models. This is because the posterior density of the model parameters is obtained by combining the prior distribution of these parameters with the likelihood function of the observed time series (Broemeling, 1985). Different types of prior distributions are employed in the Bayesian time series analysis to represent the information about the parameters of time series model. Schlaifer and Raiffa (1961) presented a natural conjugate prior that has a distributional form depends on the likelihood function form to guarantee obtaining an analytically tractable posterior distribution. In case of little or no information is available about the model parameters, Jeffreys (1961) introduced a prior, known as Jeffreys' proir, which overcomes the lack of invariance property of other existing non-informative priors. In order to simplify the elicitation of covariances of the model parameters, Zellner (1986) presented a reference informative prior known as g-prior. The availability of different types of priors makes the prior selection a complicated task and raises the issue of model identification sensitivity to prior distributions.

The identification of time series model means the model order is unknown and needs to be specified, which is is the first and crucial step in the analysis of time series models. Thus, the order of time series model can be assumed to be a random variable with a known maximum, and its posterior mass function can be derived to select the order as a value with a maximum posterior probability. Following this idea, Diaz and Farah (1981) proposed a Bayesian method to identify the order of autoregressive models. Their work has been extended by many researchers to various time series models, which include moving average models (Shaarawy et al., 2007), autoregressive moving average models 
(Fan and Yao, 2009), multivariate autoregressive models (Shaarawy and Ali, 2008), and multivariate moving average models (Shaarawy and Ali, 2012). These researchers have employed one or more of the abovementioned prior distributions to derive the posterior mass function of the model order, however, none of them has evaluated the sensitivity of model identification to different types of prior distributions. Recently, Soliman et al. (2015) have tried to evaluate the sensitivity of model identification to prior selection, however, their work is only based on empirical results and depends on a small scale of simulation study. Therefore, there is a need in the Bayesian time series analysis for a comprehensive and sophisticated evaluation of the model identification sensitivity to different priors to help researchers in the phase of prior specification.

In this paper, we use the Kullback-Leibler (KL) divergence (Kullback and Leibler, 1951) to measure the distance between the posteriors of the AR model order, resulting from different types of priors, in order to evaluate mathematically the sensitivity of the model identification to the employed priors. In addition, we evaluate the impact of the posteriors distance in the accuracy of model identification using a large number of Monte Carlo simulations. Accordingly, our work in this paper can be summarized in the following. First, we consider three types of priors for the AR model coefficient, namely Jeffreys', g, and natural conjugate priors, and also three priors for the AR model order including uniform, arithmetic, and geometric priors in order to obtain the posterior mass functions of the AR model order. Second, we compute the KL divergence and its calibration between the resulting posteriors to measure the distance between these posteriors. Third, we execute a large number of Monte Carlo simulations with various values of the model coefficients, model order, and sample size in order to evaluate the impact of the posteriors distance in the accuracy of model identification. Finally, we use real-world time series datasets to illustrate the use of the KL divergence to measure the distance between the posteriors and show the impact of this distance in the model identification.

The remainder of this paper is organized as follows. In Section 2 we present the background of the autoregressive time series model and its Bayesian concepts, and we obtain the marginal posteriors of the AR model order. In Section 3 we introduce the KL divergence and its calibration between the model order posteriors. In Section 4 we present simulation study and real-world time series datasets to illustrate the use of the KL divergence to measure the distance between the posteriors and then evaluate the impact of this distance in the model identification. Finally, we give the conclusions in Section 5.

\section{Autoregressive Time Series Models and Bayesian Concepts}

Time series $\left\{y_{t}\right\}$ can be modeled by an autoregressive (AR) model of order $p$, simply denoted by $\operatorname{AR}(p)$, and written as (Box et al., 2015):

$$
\phi_{p}(B) y_{t}=\varepsilon_{t}
$$

where $\left\{\varepsilon_{t}\right\}$ is a sequence of independent and normally distributed errors with zero mean and variance $\sigma^{2}, \mathrm{~B}$ is the backshift operator defined as $B^{d} x_{t}=x_{t-d}$, and $\phi_{p}(B)$ is the autoregressive polynomial with order $p$ written as $\phi_{p}(B)=\left(1-\phi_{1} B-\cdots-\phi_{p} B^{p}\right)$. The model (1) can be simplified and written as

$$
y=X \phi+\varepsilon,
$$


where $y=\left(y_{1}, y_{2}, \cdots, y_{n}\right)^{T}, X$ is an $n \times p$ design matrix with the $t^{\text {th }}$ row $X_{t}=$ $\left(y_{t-1}, \ldots, y_{t-p}\right), \phi=\left(\phi_{1}, \phi_{2}, \ldots, \phi_{p}\right)^{T}$ is the autoregressive coefficients, and $\varepsilon=$ $\left(\varepsilon_{1}, \varepsilon_{2}, \ldots, \varepsilon_{n}\right)^{T}$.

It is worth noting that the design matrix $X$ becomes a function of $p$ when the AR model order is unknown. In this case we can assume that the model order $p$ is a random variable with a known maximum value of $k$. The prior information about $p$ can be represented in terms of a prior mass function $\zeta(p)$ that can have different forms such as uniform, i.e. $\zeta(p)=1 / k$, or geometric, i.e. $\zeta(p)=0.5^{p} \forall p=1,2, \ldots, k$.

As we discussed above, we consider in this work three types of priors for the parameters $\phi$ and $\sigma^{2}$ : natural conjugate prior, g-prior, and Jeffreys' prior. The natural conjugate prior in the case of AR models with normally distributed errors is a normal-gamma distribution. Suppose $\phi \sim N_{p}\left(\mu_{\phi}, \sigma^{2} \Sigma_{\phi}\right)$ and $\sigma^{2} \sim I G\left(\frac{v}{2}, \frac{\lambda}{2}\right)$, the joint natural conjugate prior distribution of $\phi$ and $\sigma^{2}$ is given by:

$$
\zeta_{n}\left(\phi, \sigma^{2}\right) \propto\left(\sigma^{2}\right)^{-\left(\frac{v+p}{2}+1\right)} \exp \left\{-\frac{1}{2 \sigma^{2}}\left[\lambda+\left(\phi-\mu_{\phi}\right)^{T} \Sigma_{\phi}^{-1}\left(\phi-\mu_{\phi}\right)\right]\right\},
$$

where $\mu_{\phi}, \Sigma_{\phi}, v$ and $\lambda$ are hyperparameters need to be estimated.

The g-prior of $\phi$ and $\sigma^{2}$ can be written as:

$$
\zeta_{g}\left(\phi, \sigma^{2}\right) \propto\left(\sigma^{2}\right)^{-\left(\frac{p}{2}+1\right)} \exp \left\{-\frac{g}{2 \sigma^{2}}(\phi-\bar{\phi})^{T}\left(X^{T} X\right)(\phi-\bar{\phi})\right\},
$$

where $\bar{\phi}$ is an anticipated value of $\phi$, and $g$ is a parameter that usually specified as a decreasing function of $n$ and $p$ (Fernandez et al., 2001).

Jeffreys' prior of $\phi$ and $\sigma^{2}$ is given by:

$$
\zeta_{j}\left(\phi, \sigma^{2}\right) \propto\left(\sigma^{2}\right)^{-1}, \sigma^{2}>0
$$

The likelihood function of the AR model (2) can be obtained by employing a straightforward random variable transformation from $\varepsilon$ to $y$, and written as

$$
\begin{aligned}
& L\left(\phi, \sigma^{2}, p \mid y\right) \propto\left(\sigma^{2}\right)^{-\frac{n}{2}} \exp \left\{-\frac{1}{2 \sigma^{2}} \varepsilon^{T} \varepsilon\right\}, \\
& \propto\left(\sigma^{2}\right)^{-\frac{n}{2}} \exp \left\{-\frac{1}{2 \sigma^{2}}(y-X \phi)^{T}(y-X \phi)\right\},
\end{aligned}
$$

Based on the likelihood function (6), we update the information about the AR model order $p$ by the posterior probability mass function. To derive this posterior mass function of $p$, we need first to obtain the joint posterior of the model parameters $\phi, \sigma^{2}$ and $p$, and then integrate out the parameters $\phi$ and $\sigma^{2}$.

We obtain the joint posterior of the parameters $\phi, \sigma^{2}$ and $p$ by multiplying the likelihood function by the joint prior of these parameters. For the natural conjugate prior, the joint posterior of $\phi, \sigma^{2}$ and $p$ is obtained as:

$$
\begin{gathered}
\zeta_{n}\left(\phi, \sigma^{2}, p \mid y\right) \propto \zeta(p)\left(\sigma^{2}\right)^{-\left(\frac{n+v+p}{2}+1\right)} \exp \left\{-\frac{1}{2 \sigma^{2}}\left[\lambda+\left(\phi-\mu_{\phi}\right)^{T} \Sigma_{\phi}^{-1}\left(\phi-\mu_{\phi}\right)+\right.\right. \\
\left.\left.(y-X \phi)^{T}(y-X \phi)\right]\right\} .
\end{gathered}
$$


We integrate out the parameters $\phi$ and $\sigma^{2}$ in (7) and obtain the marginal posterior mass function of the model order $p$ as:

$$
\zeta_{n}(p \mid y) \propto \zeta(p)\left[\frac{\left|\Sigma_{\phi}^{-1}\right|}{\left|A_{n}\right|}\right]^{\frac{1}{2}}\left[y^{T} y+\lambda+\mu_{\phi}^{T} \Sigma_{\phi}^{-1} \mu_{\phi}-B_{n}^{T} A_{n}^{-1} B_{n}\right]^{-\frac{n+v}{2}} \forall p=1,2, \ldots, k .
$$

Where $A_{n}=\left(X^{T} X+\Sigma_{\phi}^{-1}\right)$ and $B_{n}=\left(X^{T} y+\Sigma_{\phi}^{-1} \mu_{\phi}\right)$

For the g-prior, we obtain the joint posterior of $\phi, \sigma^{2}$ and $p$ as:

$$
\begin{gathered}
\zeta_{g}\left(\phi, \sigma^{2}, p \mid y\right) \propto \zeta(p)\left(\sigma^{2}\right)^{-\left(\frac{n+p}{2}+1\right)} \exp \left\{-\frac{1}{2 \sigma^{2}}\left[(\phi-\bar{\phi})^{T}\left(g X^{T} X\right)(\phi-\bar{\phi})+\right.\right. \\
\left.\left.(y-X \phi)^{T}(y-X \phi)\right]\right\} .
\end{gathered}
$$

By integrating out the parameters $\phi$ and $\sigma^{2}$ in (8), we obtain the marginal posterior mass function of $p$ as:

$$
\zeta_{g}(p \mid y) \propto \zeta(p)\left[\frac{g}{g+1}\right]^{-\frac{n-p}{2}}\left[y^{T} y+g \bar{\phi}^{T}\left(X^{T} X\right) \bar{\phi}-B_{g}^{T} A_{g}^{-1} B_{g}\right]^{-\frac{n}{2}} \quad \forall p=1,2, \ldots, k .
$$

where $A_{g}=\left((g+1) X^{T} X\right)$ and $B_{g}=\left(X^{T} y+g\left(X^{T} X\right) \bar{\phi}\right)$

For Jeffreys' prior, the joint posterior of $\phi, \sigma^{2}$ and $p$ is given by :

$$
\zeta_{j}\left(\phi, \sigma^{2}, p \mid y\right) \propto \zeta(p)\left(\sigma^{2}\right)^{-\left(\frac{n}{2}+1\right)} \exp \left\{-\frac{1}{2 \sigma^{2}}(y-X \phi)^{T}(y-X \phi)\right\},
$$

Integrating out the parameters $\phi$ and $\sigma^{2}$ in (9) results in the marginal posterior mass function of $p$ as:

$$
\zeta_{j}(p \mid y) \propto \zeta(p) \frac{\Gamma\left(\frac{n-p}{2}\right)}{\pi^{\frac{n-p}{2}}\left|X^{T} X\right|^{\frac{1}{2}}}\left[y^{T} y-y^{T} X\left(X^{T} X\right)^{-1} X^{T} y\right]^{-\frac{n-p}{2}} \forall p=1,2, \ldots, k .
$$

\section{Kullback-Leibler Divergence Between Posterior Mass Functions of Model Order}

The distance between two probability mass functions, say $\zeta_{1}(x)$ and $\zeta_{2}(x)$, can be measured by the Kullback-Leibler (KL) divergence (Kullback and Leibler, 1951) defined as

$$
\begin{aligned}
K L\left[\zeta_{1}(x), \zeta_{2}(x)\right] & =E_{\zeta_{1}(x)}\left[\ln \left(\frac{\zeta_{1}(x)}{\zeta_{2}(x)}\right)\right] \\
& =\sum_{x} \zeta_{1}(x) \ln \left(\frac{\zeta_{1}(x)}{\zeta_{2}(x)}\right) .
\end{aligned}
$$

The KL divergence is always non-negative and has zero value when the two probability mass functions are identical, and the smaller its value the closer are the two probability mass functions. From that we get the motivation to evaluate the sensitivity of the AR model identification to different types of priors based on the distance between the posterior mass functions of the model order measured by the KL divergence. In particular, for two different types of priors, if the computed distance between the resulting posterior mass functions are large that implies the prior specification has an impact in the AR model identification. 
The KL divergence between two posterior mass functions of $p$, say $\zeta_{1}(p \mid y)$ and $\zeta_{2}(p \mid y)$, can be computed as:

$$
K L\left[\zeta_{1}(p \mid y), \zeta_{2}(p \mid y)\right]=\sum_{p=1}^{k} \zeta_{1}(p \mid y) \ln \left(\frac{\zeta_{1}(p \mid y)}{\zeta_{2}(p \mid y)}\right),
$$

and simplified to be:

$$
\begin{aligned}
K L\left[\zeta_{1}(p \mid y), \zeta_{2}(p \mid y)\right] & =\sum_{p=1}^{k} \zeta_{1}(p \mid y)\left[-\ln \left(\zeta_{2}(p \mid y)\right)\right]-\sum_{p=1}^{k} \zeta_{1}(p \mid y)\left[-\ln \left(\zeta_{1}(p \mid y)\right)\right] \\
& =C H\left[\zeta_{1}(p \mid y), \zeta_{2}(p \mid y)\right]-H\left[\zeta_{1}(p \mid y)\right]
\end{aligned}
$$

where the components $C H\left[\zeta_{1}(p \mid y),\left(\zeta_{2}(p \mid y)\right]\right.$ and $H\left[\zeta_{1}(p \mid y)\right]$ are known as the crossentropy and Shannon entropy respectively. The KL divergence in (12) is asymmetric measure since it does not satisfy the triangle inequality (Contreras-Reyes and ArellanoValle, 2012). However, we can get a symmetric distance by computing the average of two KL divergences as:

$$
K L^{*}\left[\zeta_{1}(p \mid y), \zeta_{2}(p \mid y)\right]=\frac{1}{2}\left\{K L\left[\zeta_{1}(p \mid y), \zeta_{2}(p \mid y)\right]+K L\left[\zeta_{2}(p \mid y), \zeta_{1}(p \mid y)\right]\right\}
$$

Values of the KL divergence are between 0 and $\infty$, and it can be calibrated to be between 0.5 and 1.0 to be easy to judge about the distance between the posteriors. Accordingly, when the calibration value of the KL divergence between two posteriors is close to 0.5 , it implies the two posteriors are very similar; and when the value is close to 1.0 the two posteriors are strongly different and the prior specification is important in this case. Suppose $K L\left[\zeta_{1}(\phi \mid y), \zeta_{2}(\phi \mid y)\right]=\gamma$, McCulloch (1989) proposed that the calibration of the KL divergence can be computed to be the value $\delta$ such that $K L[B(0.5), B(\delta)]=\gamma$, where $B(\delta)$ is a Bernoulli distribution with success probability $\delta$. Using the result that $K L[B(0.5), B(\delta)]=-\log (4 \delta(1-\delta)) / 2$ (McCulloch, 1989, Abramowitz and Stegun, 1972 ), we can compute the calibration of KL divergence (KLC) between two posteriors as:

$$
K L C\left[\zeta_{1}(\phi \mid y), \zeta_{2}(\phi \mid y)\right]=\frac{1}{2}\left\{1-\exp \left(-2 K L\left[\zeta_{1}(\phi \mid y), \zeta_{2}(\phi \mid y)\right]\right)\right\}
$$

The KL divergence in (12) and its calibration in (13) can be directly computed for any two of the marginal posteriors of $p$ presented in Section (2), and in the following section we evaluate these measures using simulated and real-world time series datasets.

\section{Application}

In this section we have two parts. In the first part, we present a simulation study to evaluate the KL divergence (and its calibration) between the posterior mass functions of the AR model order and evaluate its impact in the model identification. We present two applications of our work to real world time series datasets in the second part.

\subsection{Simulation Study}

In this section, we conduct a simulation study to evaluate the KL divergence (and its calibration) between the posterior mass functions of the AR model order, which are derived in Section (2), and evaluate the impact of this posteriors divergence in the model 
identification. We generate several time series data with considering different sample size, different model orders, and different values of the model coefficients.

For the prior specification in this simulation study, we employ the uniform prior for the model order $p$ with a known maximum $k=4$. In addition, we use three values for the parameter $g$, i.e. $1 / n, p / n$, and $k^{p} / n$, for the g-prior, following the recommendations of Fernandez et al. (2001), and also we follow the training sample approach to estimate the hyperparamters of the natural conjugate prior (Berger, 1985, Rachev et al., 2008). To run the simulations, we generate 1,000 time series of size $n$ (from 50 to 400 with an increment of 50 observations) from AR models with orders $1(\phi=0.3,0.5$, and 0.8$), 2$ $\left(\phi_{1}=0.5\right.$ and 0.2 , and $\phi_{2}=0.4$ and 0.6$)$, and $3\left(\phi_{1}=0.5\right.$ and $0.2, \phi_{2}=0.4$ and 0.6 , and $\phi_{3}=0.4$ and 0.6 ). For each time series, we compute the posterior mass functions of $p$, $\zeta_{j}(p \mid y), \zeta_{g}(p \mid y)$, and $\zeta_{n}(p \mid y)$ resulting from the employed priors Jeffreys', g, and natural conjugate respectively. Based on the computed posterior mass functions of $p$, we compute the KL divergence (and its calibration) between these posteriors, and identify the model order as a value with a maximum posterior probability. For all simulated time series, we compute the percentage of correctly identified models by comparing the identified order with the true value of $p$ used to generate the time series.

We present the average of KL divergence and its calibration obtained with the 1,000 time series for $\operatorname{AR}(1)$ with $\phi=0.3$ in Table (1) and particularly the boxplot of the KL calibration (for $n=200$ ) in Figure (1), and present the percentage of correctly identified models in Table (2). From these results, we observe that the KL divergence and its calibration between $\zeta_{j}(p \mid y)$ and $\zeta_{g 1}(p \mid y)$ and those between $\zeta_{j}(p \mid y)$ and $\zeta_{g 3}(p \mid y)$ are very close, for example when $n=200$ their calibration values are about 0.73 . These divergences are strongly larger than those between $\zeta_{j}(p \mid y)$ and $\zeta_{g 2}(p \mid y)$ and those between $\zeta_{j}(p \mid y)$ and $\zeta_{n}(p \mid y)$, which both have calibration values of about 0.6 for $n=$ 200 as an example. The impact of these posteriors divergences can be observed in the percentage of correctly identified models presented in Table (1), since the results show that the highest percentage of correctly identified models is obtained from the two posteriors resulting from the g-prior with $g=1 / n$ and $k^{p} / n$ followed by the percentage obtained from the two posteriors resulting from the g-prior with $g=p / n$ and from the natural conjugate prior, and the lowest percentage is obtained from the posterior resulting from the Jeffreys' prior. It is worth observing that when the sample size grows the divergences of posteriors mass functions are reduced. For large sample size $(n=400)$, all the KL calibration values are less than 0.7 , and all the percentages of correctly identified models are greater than 95\%. This confirms that the impact of the prior distribution will be eliminated for a large sample of observations.

We get approximately same results from $\operatorname{AR}(2)$ and $\operatorname{AR}(3)$ models with an additional observation that the more complicated AR model the smaller percentage of correctly identified models, especially in case of small sample size. In particular, the results of AR(2) model with $\phi_{1}=0.2$ and $\phi_{2}=0.6$ are presented in Tables (3) and (4), and also the results of $\operatorname{AR}(3)$ model with $\phi_{1}=0.5, \phi_{2}=-0.4$ and $\phi_{3}=0.6$ are presented in Tables (5) and (6). 
Table 1: KL divergence and its calibration for $\operatorname{AR}(1)$ with $\phi=0.3$

\begin{tabular}{|c|c|c|c|c|c|c|c|c|}
\hline \multicolumn{9}{|c|}{ Sample size $(n)$} \\
\hline & 50 & 100 & 150 & 200 & 250 & 300 & 350 & 400 \\
\hline$K L\left[\zeta_{j}, \zeta_{g 1}\right]^{*}$ & 0.322 & 0.211 & 0.164 & 0.134 & 0.122 & 0.109 & 0.101 & 0.093 \\
\hline$K L C\left[\zeta_{j}, \zeta_{g 1}\right]$ & 0.837 & 0.784 & 0.755 & 0.734 & 0.723 & 0.711 & 0.704 & 0.696 \\
\hline$K L\left[\zeta_{j}, \zeta_{g 2}\right]$ & 0.068 & 0.038 & 0.028 & 0.023 & 0.021 & 0.019 & 0.016 & 0.015 \\
\hline$K L C\left[\zeta_{j}, \zeta_{g 2}\right]$ & 0.655 & 0.619 & 0.603 & 0.594 & 0.589 & 0.584 & 0.58 & 0.577 \\
\hline$K L\left[\zeta_{j}, \zeta_{g 3}\right]$ & 0.315 & 0.207 & 0.161 & 0.13 & 0.119 & 0.106 & 0.099 & 0.091 \\
\hline$K L C\left[\zeta_{j}, \zeta_{g 3}\right]$ & 0.833 & 0.78 & 0.751 & 0.73 & 0.719 & 0.708 & 0.7 & 0.693 \\
\hline$K L\left[\zeta_{j}, \zeta_{n}\right]$ & 0.112 & 0.067 & 0.052 & 0.039 & 0.037 & 0.032 & 0.029 & 0.026 \\
\hline$K L C\left[\zeta_{j}, \zeta_{n}\right]$ & 0.667 & 0.631 & 0.615 & 0.604 & 0.599 & 0.593 & 0.589 & 0.585 \\
\hline$K L\left[\zeta_{g 1}, \zeta_{n}\right]$ & 0.19 & 0.147 & 0.126 & 0.107 & 0.1 & 0.09 & 0.085 & 0.081 \\
\hline$K L C\left[\zeta_{g 1}, \zeta_{n}\right]$ & 0.754 & 0.728 & 0.711 & 0.697 & 0.69 & 0.681 & 0.675 & 0.67 \\
\hline
\end{tabular}

${ }^{*} \zeta_{g 1}, \zeta_{g 2}$, and $\zeta_{g 3}$ posteriors from g-prior with $g=1 / n, p / n$ and $k^{p} / n$ respectively, and $\zeta_{j}$ and $\zeta_{n}$ posteriors from Jeffreys' and natural conjugate priors respectively.

Table 2: Percentage of correctly identified models for $\operatorname{AR}(1)$ with $\phi=0.3$.

\begin{tabular}{|c|c|c|c|c|c|c|c|c|}
\hline \multicolumn{9}{|c|}{ Sample size (n) } \\
\hline & $\mathbf{5 0}$ & $\mathbf{1 0 0}$ & $\mathbf{1 5 0}$ & $\mathbf{2 0 0}$ & $\mathbf{2 5 0}$ & $\mathbf{3 0 0}$ & $\mathbf{3 5 0}$ & $\mathbf{4 0 0}$ \\
\hline $\boldsymbol{\zeta}_{\boldsymbol{j}}(\boldsymbol{p} \mid \boldsymbol{y})$ & 76.6 & 87.2 & 90.8 & 92.9 & 92.9 & 95 & 94.9 & 95.8 \\
\hline $\boldsymbol{\zeta}_{\boldsymbol{g} \mathbf{1}}(\boldsymbol{p} \mid \boldsymbol{y})$ & 93.9 & 97.1 & 97.9 & 98.4 & 98.6 & 98.6 & 97.8 & 98.3 \\
\hline $\boldsymbol{\zeta}_{\boldsymbol{g} \mathbf{2}}(\boldsymbol{p} \mid \boldsymbol{y})$ & 87.2 & 92.5 & 94.7 & 95.2 & 94.9 & 96.6 & 96.3 & 97.2 \\
\hline $\boldsymbol{\zeta}_{\boldsymbol{g} \mathbf{3}}(\boldsymbol{p} \mid \boldsymbol{y})$ & 94.3 & 96.8 & 98 & 98.3 & 98.3 & 98.5 & 97.8 & 98.3 \\
\hline $\boldsymbol{\zeta}_{\boldsymbol{n}}(\boldsymbol{p} \mid \boldsymbol{y})$ & 84.9 & 89.9 & 92.3 & 94 & 93.8 & 95.6 & 95.6 & 95.9 \\
\hline
\end{tabular}




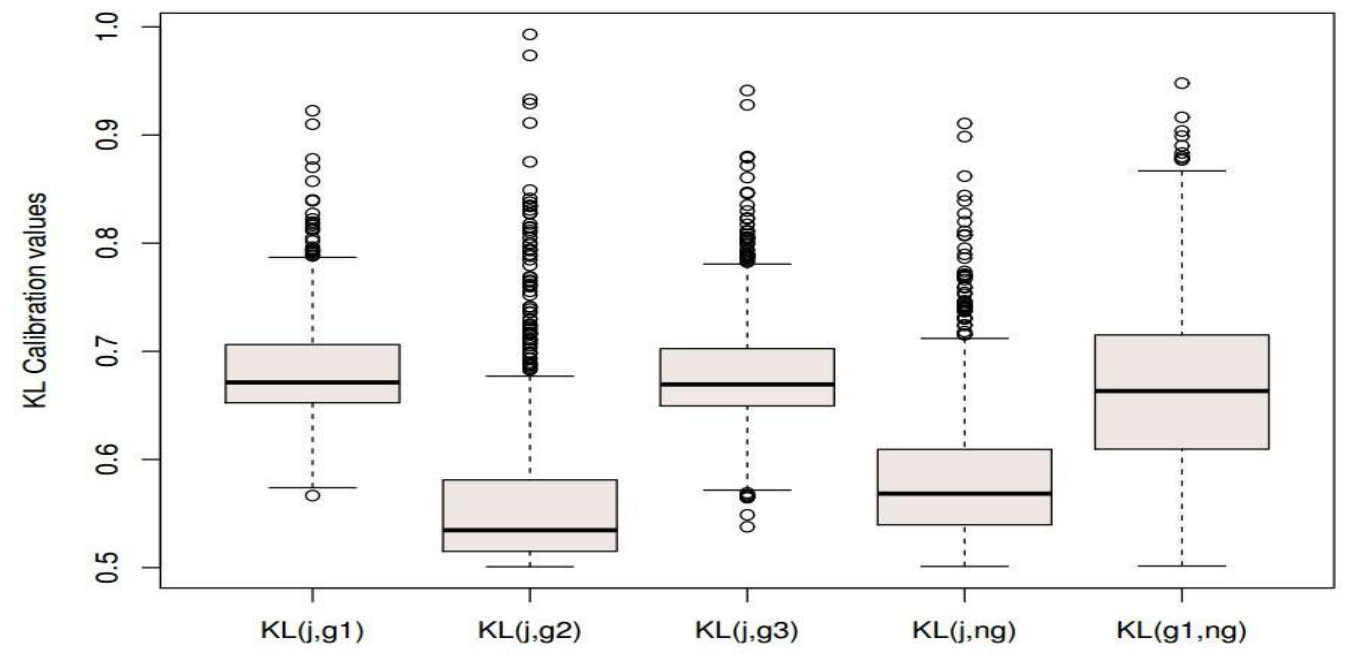

Figure 1: Boxplot of the KL calibration for AR(1) with $\phi=0.3$ and $n=200$

Table 3: KL divergence and its calibration for $\operatorname{AR}(2)$ with $\phi_{1}=0.2$ and $\phi_{2}=0.6$

\begin{tabular}{|c|c|c|c|c|c|c|c|c|}
\hline \multicolumn{10}{|c|}{} & $\mathbf{5 0}$ & $\mathbf{1 0 0}$ & $\mathbf{1 5 0}$ & $\mathbf{2 0 0}$ & $\mathbf{2 5 0}$ & $\mathbf{3 0 0}$ & $\mathbf{3 5 0}$ & $\mathbf{4 0 0}$ \\
\hline $\boldsymbol{K} \boldsymbol{L}\left[\boldsymbol{\zeta}_{\boldsymbol{j}}, \boldsymbol{\zeta}_{\boldsymbol{g} \mathbf{1}}\right]$ & 0.229 & 0.155 & 0.128 & 0.11 & 0.1 & 0.092 & 0.086 & 0.08 \\
\hline $\boldsymbol{K} \boldsymbol{L}\left[\boldsymbol{\zeta}_{\boldsymbol{j}}, \boldsymbol{\zeta}_{\boldsymbol{g} \mathbf{1}}\right]$ & 0.797 & 0.752 & 0.73 & 0.715 & 0.705 & 0.697 & 0.69 & 0.684 \\
\hline $\boldsymbol{K} \boldsymbol{L}\left[\boldsymbol{\zeta}_{\boldsymbol{j}}, \boldsymbol{\zeta}_{\boldsymbol{g} \mathbf{2}}\right]$ & 0.071 & 0.05 & 0.043 & 0.033 & 0.031 & 0.028 & 0.027 & 0.024 \\
\hline $\boldsymbol{K} \boldsymbol{L} \boldsymbol{C}\left[\boldsymbol{\zeta}_{\boldsymbol{j}}, \boldsymbol{\zeta}_{\boldsymbol{g} \mathbf{2}}\right]$ & 0.62 & 0.592 & 0.582 & 0.574 & 0.57 & 0.567 & 0.564 & 0.561 \\
\hline $\boldsymbol{K} \boldsymbol{L}\left[\boldsymbol{\zeta}_{\boldsymbol{j}}, \boldsymbol{\zeta}_{\boldsymbol{g} \mathbf{3}}\right]$ & 0.224 & 0.154 & 0.127 & 0.109 & 0.099 & 0.091 & 0.085 & 0.079 \\
\hline $\boldsymbol{K} \boldsymbol{L}\left[\boldsymbol{\zeta}_{\boldsymbol{j}}, \boldsymbol{\zeta}_{\boldsymbol{g} \mathbf{3}}\right]$ & 0.792 & 0.749 & 0.727 & 0.712 & 0.702 & 0.694 & 0.687 & 0.681 \\
\hline $\boldsymbol{K} \boldsymbol{L}\left[\boldsymbol{\zeta}_{\boldsymbol{j}}, \boldsymbol{\zeta}_{\boldsymbol{n}}\right]$ & 0.089 & 0.056 & 0.046 & 0.037 & 0.035 & 0.031 & 0.028 & 0.026 \\
\hline $\boldsymbol{K} \boldsymbol{L} \boldsymbol{C}\left[\boldsymbol{\zeta}_{\boldsymbol{j}}, \boldsymbol{\zeta}_{\boldsymbol{n}}\right]$ & 0.650 & 0.618 & 0.605 & 0.598 & 0.593 & 0.59 & 0.586 & 0.583 \\
\hline $\boldsymbol{K} \boldsymbol{L}\left[\boldsymbol{\zeta}_{\boldsymbol{g} \mathbf{1}}, \boldsymbol{\zeta}_{\boldsymbol{n}}\right]$ & 0.167 & 0.115 & 0.101 & 0.091 & 0.084 & 0.08 & 0.074 & 0.07 \\
\hline $\boldsymbol{K} \boldsymbol{L} \boldsymbol{C}\left[\boldsymbol{\zeta}_{\boldsymbol{g} \mathbf{1}}, \boldsymbol{\zeta}_{\boldsymbol{n}}\right]$ & 0.736 & 0.704 & 0.691 & 0.681 & 0.675 & 0.67 & 0.665 & 0.66 \\
\hline
\end{tabular}

Table 4: Percentage of correctly identified models for $\operatorname{AR}(2)$ with $\phi_{1}=0.2$ and $\phi_{2}=0.6$.

\begin{tabular}{|c|c|c|c|c|c|c|c|c|}
\hline \multicolumn{9}{|c|}{ Sample size (n) } \\
\hline & $\mathbf{5 0}$ & $\mathbf{1 0 0}$ & $\mathbf{1 5 0}$ & $\mathbf{2 0 0}$ & $\mathbf{2 5 0}$ & $\mathbf{3 0 0}$ & $\mathbf{3 5 0}$ & $\mathbf{4 0 0}$ \\
\hline $\boldsymbol{\zeta}_{\boldsymbol{j}}(\boldsymbol{p} \mid \boldsymbol{y})$ & 80.4 & 88.9 & 91.9 & 94.5 & 93.8 & 94.6 & 94.3 & 95.3 \\
\hline $\boldsymbol{\zeta}_{\boldsymbol{g} \mathbf{1}}(\boldsymbol{p} \mid \boldsymbol{y})$ & 90.6 & 97.4 & 97.4 & 98.3 & 97.8 & 98.7 & 98.4 & 98.9 \\
\hline $\boldsymbol{\zeta}_{\boldsymbol{g} \mathbf{2}}(\boldsymbol{p} \mid \boldsymbol{y})$ & 85.7 & 91.6 & 94.1 & 95 & 95.1 & 95.8 & 95.1 & 95.6 \\
\hline $\boldsymbol{\zeta}_{\boldsymbol{g} \mathbf{3}}(\boldsymbol{p} \mid \boldsymbol{y})$ & 91.4 & 97.2 & 97.1 & 98.3 & 97.6 & 98.7 & 98.2 & 98.9 \\
\hline $\boldsymbol{\zeta}_{\boldsymbol{n}}(\boldsymbol{p} \mid \boldsymbol{y})$ & 84.6 & 90.8 & 92.4 & 94.5 & 94.1 & 94.9 & 94.6 & 95.1 \\
\hline
\end{tabular}


Table 5: KL divergence and its calibration for $\operatorname{AR}(3)$ with $\phi_{1}=0.5, \phi_{2}=-0.4$ and $\phi_{3}=0.6$.

\begin{tabular}{|c|c|c|c|c|c|c|c|c|}
\hline \multicolumn{10}{|c|}{ Sample size $(\boldsymbol{n})$} \\
\hline & $\mathbf{5 0}$ & $\mathbf{1 0 0}$ & $\mathbf{1 5 0}$ & $\mathbf{2 0 0}$ & $\mathbf{2 5 0}$ & $\mathbf{3 0 0}$ & $\mathbf{3 5 0}$ & $\mathbf{4 0 0}$ \\
\hline $\boldsymbol{K} \boldsymbol{L}\left[\boldsymbol{\zeta}_{\boldsymbol{j}}, \boldsymbol{\zeta}_{\boldsymbol{g} \mathbf{1}}\right]$ & 0.151 & 0.081 & 0.07 & 0.063 & 0.059 & 0.056 & 0.053 & 0.051 \\
\hline $\boldsymbol{K} \boldsymbol{L} \boldsymbol{C}\left[\boldsymbol{\zeta}_{\boldsymbol{j}}, \boldsymbol{\zeta}_{\boldsymbol{g} \mathbf{1}}\right]$ & 0.739 & 0.69 & 0.677 & 0.668 & 0.662 & 0.658 & 0.654 & 0.65 \\
\hline $\boldsymbol{K} \boldsymbol{L}\left[\boldsymbol{\zeta}_{\boldsymbol{j}}, \boldsymbol{\zeta}_{\boldsymbol{g} \mathbf{2}}\right]$ & 0.054 & 0.035 & 0.03 & 0.027 & 0.025 & 0.023 & 0.021 & 0.02 \\
\hline $\boldsymbol{K} \boldsymbol{L} \boldsymbol{C}\left[\boldsymbol{\zeta}_{\boldsymbol{j}}, \boldsymbol{\zeta}_{\boldsymbol{g} \mathbf{2}}\right]$ & 0.585 & 0.565 & 0.56 & 0.558 & 0.555 & 0.554 & 0.553 & 0.552 \\
\hline $\boldsymbol{K} \boldsymbol{L}\left[\boldsymbol{\zeta}_{\boldsymbol{j}}, \boldsymbol{\zeta}_{\boldsymbol{g} \mathbf{3}}\right]$ & 0.147 & 0.082 & 0.071 & 0.064 & 0.059 & 0.056 & 0.053 & 0.051 \\
\hline $\boldsymbol{K} \boldsymbol{L C}\left[\boldsymbol{\zeta}_{\boldsymbol{j}}, \boldsymbol{\zeta}_{\boldsymbol{g} \mathbf{3}}\right]$ & 0.736 & 0.689 & 0.676 & 0.667 & 0.661 & 0.657 & 0.653 & 0.649 \\
\hline $\boldsymbol{K} \boldsymbol{L}\left[\boldsymbol{\zeta}_{\boldsymbol{j}}, \boldsymbol{\zeta}_{\boldsymbol{n}}\right]$ & 0.054 & 0.031 & 0.025 & 0.021 & 0.02 & 0.017 & 0.016 & 0.015 \\
\hline $\boldsymbol{K} \boldsymbol{L} \boldsymbol{C}\left[\boldsymbol{\zeta}_{\boldsymbol{j}}, \boldsymbol{\zeta}_{\boldsymbol{n}}\right]$ & 0.613 & 0.583 & 0.574 & 0.569 & 0.566 & 0.564 & 0.562 & 0.56 \\
\hline $\boldsymbol{K} \boldsymbol{L}\left[\boldsymbol{\zeta}_{\boldsymbol{g} \mathbf{1}}, \boldsymbol{\zeta}_{\boldsymbol{n}}\right]$ & 0.131 & 0.061 & 0.053 & 0.048 & 0.047 & 0.044 & 0.042 & 0.04 \\
\hline$K \boldsymbol{L} C\left[\zeta_{\boldsymbol{g} 1}, \boldsymbol{\zeta}_{\boldsymbol{n}}\right]$ & 0.697 & 0.648 & 0.64 & 0.635 & 0.631 & 0.628 & 0.625 & 0.623 \\
\hline
\end{tabular}

Table 6: Percentage of correctly identified models for $\operatorname{AR}(3)$ with $\phi_{1}=0.5, \phi_{2}=$ -0.4 and $\phi_{3}=0.6$.

\begin{tabular}{|c|c|c|c|c|c|c|c|c|}
\hline \multicolumn{9}{|c|}{ Sample size (n) } \\
\hline & $\mathbf{5 0}$ & $\mathbf{1 0 0}$ & $\mathbf{1 5 0}$ & $\mathbf{2 0 0}$ & $\mathbf{2 5 0}$ & $\mathbf{3 0 0}$ & $\mathbf{3 5 0}$ & $\mathbf{4 0 0}$ \\
\hline $\boldsymbol{\zeta}_{\boldsymbol{j}}(\boldsymbol{p} \mid \boldsymbol{y})$ & 84.7 & 89.4 & 91.7 & 94.5 & 94.2 & 95.3 & 95.8 & 95.6 \\
\hline $\boldsymbol{\zeta}_{\boldsymbol{g} \mathbf{1}}(\boldsymbol{p} \mid \boldsymbol{y})$ & 89.6 & 97.2 & 98.0 & 98.3 & 98.4 & 99.1 & 98.7 & 99.1 \\
\hline $\boldsymbol{\zeta}_{\boldsymbol{g} \mathbf{2}}(\boldsymbol{p} \mid \boldsymbol{y})$ & 86.8 & 91.6 & 93.3 & 95.4 & 94.8 & 95.5 & 95.6 & 96.1 \\
\hline $\boldsymbol{\zeta}_{\boldsymbol{g} \mathbf{3}}(\boldsymbol{p} \mid \boldsymbol{y})$ & 90.3 & 97.1 & 98.1 & 98.3 & 98.3 & 98.9 & 98.5 & 99.0 \\
\hline $\boldsymbol{\zeta}_{\boldsymbol{n}}(\boldsymbol{p} \mid \boldsymbol{y})$ & 89.1 & 92.4 & 93.9 & 95.9 & 95.7 & 96.2 & 96.1 & 96.6 \\
\hline
\end{tabular}

Now, one question can be raised: is there an impact for the prior distribution of the model order in the posteriors divergence and the percentage of correctly identified models? To answer this question we modify the simulation design to employ only the g-prior with $g=1 / n$ for the coefficients $\phi$, and employ three prior distributions for $p$ given as:

$$
\begin{aligned}
& \zeta_{1}(p)=\frac{1}{k}, \forall p=1,2, \ldots, k \quad \text { (Uniform prior) } \\
& \zeta_{2}(p)=0.5^{p}, \forall p=1,2, \ldots, k \quad \text { (Geometric prior) } \\
& \zeta_{3}(p)=\frac{k-p+1}{k+1}, \forall p=1,2, \ldots, k \quad \text { (Arithmetic prior) }
\end{aligned}
$$

Results of AR(1) with $\phi=0.3$, presented in Tables (7) and (8), show that the posterior mass functions of $p$ resulting from the employed three priors in (3) are slightly different and their divergence impact in the percentage of correctly identified models is small and can be ignored especially for sample size $n \geq 100$. We get approximately same results from $\operatorname{AR}(2)$ model with $\phi_{1}=0.2$ and $\phi_{2}=0.6$, presented in Tables (9) and (10), and from AR(3) model with $\phi_{1}=0.5, \phi_{2}=-0.4$ and $\phi_{3}=0.6$, presented in Tables (11) and (12). 
In general, all simulation results show that the employed priors for the model order result in very similar posteriors. However, the posteriors of the model order resulting from the employed priors for the model coefficients are strongly different, and the highest percentage of identified models is obtained from the posterior resulting from the g-prior distribution.

Table 7: KL divergence and its calibration for $\operatorname{AR}(1)$ with $\phi=0.3$

\begin{tabular}{|c|c|c|c|c|c|c|c|c|}
\hline \multicolumn{9}{|c|}{ Sample size $(n)$} \\
\hline & 50 & 100 & 150 & 200 & 250 & 300 & 350 & 400 \\
\hline$K L\left[\zeta_{1}, \zeta_{2}\right]^{*}$ & 0.059 & 0.04 & 0.032 & 0.026 & 0.024 & 0.021 & 0.02 & 0.018 \\
\hline$K L C\left[\zeta_{1}, \zeta_{2}\right]$ & 0.66 & 0.632 & 0.618 & 0.607 & 0.602 & 0.596 & 0.593 & 0.59 \\
\hline$K L\left[\zeta_{1}, \zeta_{3}\right]$ & 0.019 & 0.012 & 0.009 & 0.007 & 0.006 & 0.005 & 0.005 & 0.005 \\
\hline$K L C\left[\zeta_{1}, \zeta_{3}\right]$ & 0.59 & 0.57 & 0.561 & 0.555 & 0.552 & 0.548 & 0.547 & 0.545 \\
\hline$K L\left[\zeta_{2}, \zeta_{3}\right]$ & 0.013 & 0.009 & 0.008 & 0.007 & 0.006 & 0.005 & 0.005 & 0.005 \\
\hline$K L C\left[\zeta_{2}, \zeta_{3}\right]$ & 0.577 & 0.565 & 0.56 & 0.555 & 0.552 & 0.549 & 0.548 & 0.546 \\
\hline
\end{tabular}

${ }^{*} \zeta_{1}, \zeta_{2}$, and $\zeta_{3}$ are posteriors from priors $\zeta_{1}(p), \zeta_{2}(p)$, and $\zeta_{3}(p)$, respectively.

Table 8: Percentage of correctly identified models for $\operatorname{AR}(1)$ with $\phi=0.3$.

\begin{tabular}{|c|c|c|c|c|c|c|c|c|}
\hline \multicolumn{9}{|c|}{ Sample size (n) } \\
\hline & $\mathbf{5 0}$ & $\mathbf{1 0 0}$ & $\mathbf{1 5 0}$ & $\mathbf{2 0 0}$ & $\mathbf{2 5 0}$ & $\mathbf{3 0 0}$ & $\mathbf{3 5 0}$ & $\mathbf{4 0 0}$ \\
\hline $\boldsymbol{\zeta}_{\mathbf{1}}(\boldsymbol{p} \mid \boldsymbol{y})$ & 93.9 & 97.1 & 97.9 & 98.4 & 98.6 & 98.6 & 97.8 & 98.3 \\
\hline $\boldsymbol{\zeta}_{\mathbf{2}}(\boldsymbol{p} \mid \boldsymbol{y})$ & 97.6 & 98.9 & 99.2 & 99.5 & 99.5 & 99.1 & 98.9 & 99.1 \\
\hline $\boldsymbol{\zeta}_{\mathbf{3}}(\boldsymbol{p} \mid \boldsymbol{y})$ & 96.1 & 98.3 & 98.9 & 99.2 & 99 & 98.9 & 98.6 & 98.5 \\
\hline
\end{tabular}

Table 9: KL divergence and its calibration for $\mathrm{AR}(2)$ with $\phi_{1}=0.2$ and $\phi_{2}=0.6$.

\begin{tabular}{|c|c|c|c|c|c|c|c|c|}
\hline \multicolumn{9}{|c|}{ Sample size $(n)$} \\
\hline & 50 & 100 & 150 & 200 & 250 & 300 & 350 & 400 \\
\hline$K L\left[\zeta_{1}, \zeta_{2}\right]$ & 0.056 & 0.034 & 0.027 & 0.024 & 0.022 & 0.02 & 0.019 & 0.018 \\
\hline$K L C\left[\zeta_{1}, \zeta_{2}\right.$ & 0.657 & 0.623 & 0.61 & 0.603 & 0.598 & 0.594 & 0.591 & 0.588 \\
\hline$K L\left[\zeta_{1}, \zeta_{3}\right]$ & 0.025 & 0.017 & 0.013 & 0.011 & 0.01 & 0.009 & 0.009 & 0.008 \\
\hline$K L C\left[\zeta_{1}, \zeta_{3}\right.$ & 0.607 & 0.586 & 0.577 & 0.571 & 0.567 & 0.564 & 0.562 & 0.559 \\
\hline$K L\left[\zeta_{2}, \zeta_{3}\right]$ & 0.008 & 0.004 & 0.003 & 0.003 & 0.002 & 0.002 & 0.002 & 0.002 \\
\hline$K L C\left[\zeta_{2}, \zeta_{3}\right.$ & 0.559 & 0.541 & 0.537 & 0.535 & 0.533 & 0.532 & 0.531 & 0.531 \\
\hline
\end{tabular}


Table 10: Percentage of correctly identified models for $\operatorname{AR}(2)$ with $\phi_{1}=0.2$ and $\phi_{2}=0.6$.

\begin{tabular}{|c|c|c|c|c|c|c|c|c|}
\hline \multicolumn{10}{|c|}{ Sample size (n) } \\
\hline & $\mathbf{5 0}$ & $\mathbf{1 0 0}$ & $\mathbf{1 5 0}$ & $\mathbf{2 0 0}$ & $\mathbf{2 5 0}$ & $\mathbf{3 0 0}$ & $\mathbf{3 5 0}$ & $\mathbf{4 0 0}$ \\
\hline $\boldsymbol{\zeta}_{\mathbf{1}}(\boldsymbol{p} \mid \boldsymbol{y})$ & 90.6 & 97.4 & 97.4 & 98.3 & 97.8 & 98.7 & 98.4 & 98.9 \\
\hline $\boldsymbol{\zeta}_{\mathbf{2}}(\boldsymbol{p} \mid \boldsymbol{y})$ & 91.1 & 98.7 & 98.8 & 99.3 & 98.9 & 99.5 & 99.4 & 99.6 \\
\hline $\boldsymbol{\zeta}_{\mathbf{3}}(\boldsymbol{p} \mid \boldsymbol{y})$ & 91.5 & 98.4 & 98.1 & 99.1 & 98.5 & 99.3 & 99.2 & 99.4 \\
\hline
\end{tabular}

Table 11: KL divergence and its calibration for $\operatorname{AR}(3)$ with $\phi_{1}=0.5, \phi_{2}=-0.4$ and $\phi_{3}=0.6$.

\begin{tabular}{|c|c|c|c|c|c|c|c|c|}
\hline \multicolumn{9}{|c|}{ Sample size $(n)$} \\
\hline & 50 & 100 & 150 & 200 & 250 & 300 & 350 & 400 \\
\hline$K L\left[\zeta_{1}, \zeta_{2}\right]$ & 0.071 & 0.025 & 0.019 & 0.017 & 0.016 & 0.015 & 0.014 & 0.014 \\
\hline$K L C\left[\zeta_{1}, \zeta_{2}\right]$ & 0.662 & 0.605 & 0.595 & 0.589 & 0.586 & 0.583 & 0.581 & 0.578 \\
\hline$K L\left[\zeta_{1}, \zeta_{3}\right]$ & 0.037 & 0.023 & 0.019 & 0.017 & 0.016 & 0.015 & 0.014 & 0.014 \\
\hline$K L C\left[\zeta_{1}, \zeta_{3}\right]$ & 0.629 & 0.603 & 0.595 & 0.589 & 0.586 & 0.583 & 0.581 & 0.578 \\
\hline$K L\left[\zeta_{2}, \zeta_{3}\right]$ & 0.011 & 0.001 & 0.000 & 0.000 & 0.000 & 0.000 & 0.000 & 0.000 \\
\hline$K L C\left[\zeta_{2}, \zeta_{3}\right]$ & 0.549 & 0.504 & 0.500 & 0.500 & 0.500 & 0.500 & 0.500 & 0.500 \\
\hline
\end{tabular}

Table 12: Percentage of correctly identified models for $\operatorname{AR}(3)$ with $\phi_{1}=0.5$, $\phi_{2}=-0.4$ and $\phi_{3}=0.6$.

\begin{tabular}{|c|c|c|c|c|c|c|c|c|}
\hline \multicolumn{10}{|c|}{ Sample size (n) } \\
\hline & $\mathbf{5 0}$ & $\mathbf{1 0 0}$ & $\mathbf{1 5 0}$ & $\mathbf{2 0 0}$ & $\mathbf{2 5 0}$ & $\mathbf{3 0 0}$ & $\mathbf{3 5 0}$ & $\mathbf{4 0 0}$ \\
\hline $\boldsymbol{\zeta}_{\mathbf{1}}(\boldsymbol{p} \mid \boldsymbol{y})$ & 89.6 & 97.2 & 98 & 98.3 & 98.4 & 99.1 & 98.7 & 99.1 \\
\hline $\boldsymbol{\zeta}_{\mathbf{2}}(\boldsymbol{p} \mid \boldsymbol{y})$ & 85.7 & 98 & 99.1 & 99.5 & 99.6 & 99.6 & 99.6 & 99.6 \\
\hline $\boldsymbol{\zeta}_{\mathbf{3}}(\boldsymbol{p} \mid \boldsymbol{y})$ & 88.9 & 98.2 & 99.1 & 99.5 & 99.6 & 99.6 & 99.6 & 99.6 \\
\hline
\end{tabular}

\subsection{Application to Real-World Time Series}

In this subsection, we use four real-world time series datasets to illustrate the use of the KL divergence and its calibration to measure the distance between the posterior mass functions of the model order, and show the impact of this distance in the model identification. These real-world time series datasets are described in Table (13) and plotted in Figure (2). 
Table 13: Description of the real-world time series datasets (Box et al., 2015)

\begin{tabular}{|c|l|c|}
\hline Series & \multicolumn{1}{|c|}{ Description } & Sample size \\
\hline A & $\begin{array}{l}\text { Readings of chemical process temperature are collected every } \\
\text { minute by temporarily disconnecting the controllers and } \\
\text { recording the subsequent fluctuations in temperature. }\end{array}$ & 226 \\
\hline B & $\begin{array}{l}\text { Readings of chemical process viscosity are collected every hour } \\
\text { to show the effect of uncontrolled factors such as variations in } \\
\text { ambient temperature. }\end{array}$ & 310 \\
\hline C & $\begin{array}{l}\text { Numbers of annual Wolfer sunspot are collected over the period } \\
1770-1869 .\end{array}$ & 100 \\
\hline D & $\begin{array}{l}\text { Yields are collected from consecutive batches of a chemical } \\
\text { process. }\end{array}$ & 70 \\
\hline
\end{tabular}

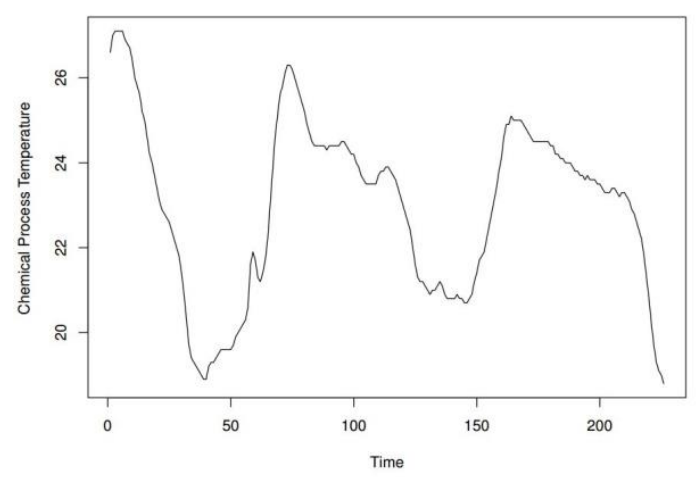

(a) Chemical process temperature readings

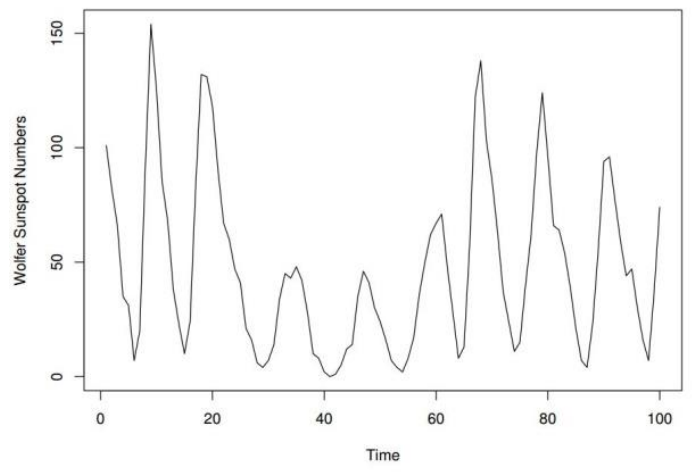

(c) Annual Wolfer sunspot numbers

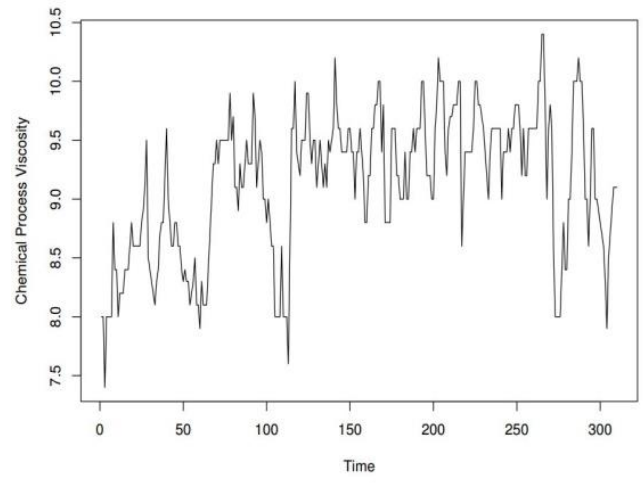

(b) Chemical process viscosity readings

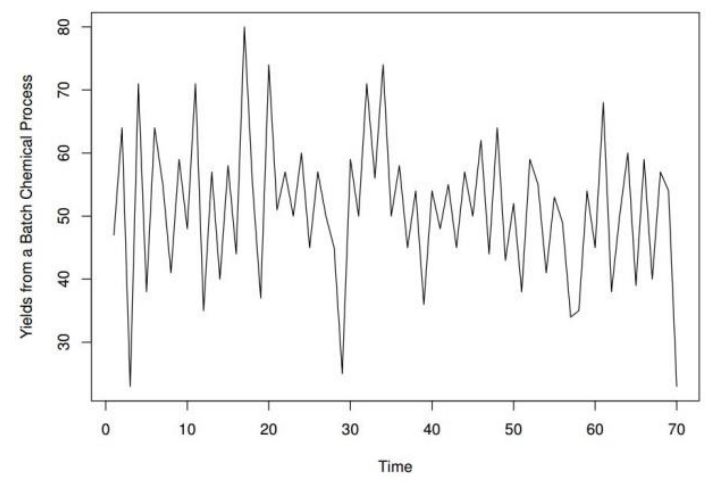

(d) Yields from chemical process batches

Figure 2: Time-plot of the real-world time series datasets 
Using the non-Bayesian approach, Box et al. (2015) identified AR(1) model for the time series $\mathrm{A}$ and $\mathrm{B}$, and identified $\mathrm{AR}(2)$ model for the time series $\mathrm{C}$ and $\mathrm{D}$. We first employ Jeffreys', g, and natural conjugate priors to obtain the posteriors of the model order. Second, we compute the KL divergence and its calibration between these posteriors. Finally, we compute the posterior probabilities and identify the model order with the maximum posterior probability. Results of the KL divergence and its calibration presented in Table (14) and results of the posterior probability and identified model are presented in Table (15). It can be observed that these results are very consistent with the results from the simulation study in previous subsection, and the identified models are almost the same as those identified by the non-Bayesian approach.

Table 14: KL divergence and its calibration for the real-world datasets.

\begin{tabular}{|c|c|c|c|c|}
\hline & \multicolumn{4}{|c|}{ Real-world time series } \\
\hline & $\mathbf{A}$ & $\mathbf{B}$ & $\mathbf{C}$ & $\mathbf{D}$ \\
\hline $\boldsymbol{K} \boldsymbol{L}\left[\boldsymbol{\zeta}_{\boldsymbol{j}}, \boldsymbol{\zeta}_{\boldsymbol{g} \mathbf{1}}\right]$ & 0.081 & 0.175 & 0.269 & 0.358 \\
\hline $\boldsymbol{K} \boldsymbol{L}\left[\boldsymbol{\zeta}_{\boldsymbol{j}}, \boldsymbol{\zeta}_{\boldsymbol{g} \mathbf{1}}\right]$ & 0.693 & 0.772 & 0.823 & 0.858 \\
\hline $\boldsymbol{K} \boldsymbol{L}\left[\boldsymbol{\zeta}_{\boldsymbol{j}}, \boldsymbol{\zeta}_{\boldsymbol{g} \mathbf{2}}\right]$ & 0.007 & 0.05 & 0.001 & 0.06 \\
\hline $\boldsymbol{K} \boldsymbol{L} \boldsymbol{C}\left[\boldsymbol{\zeta}_{\boldsymbol{j}}, \boldsymbol{\zeta}_{\boldsymbol{g} \mathbf{2}}\right]$ & 0.56 & 0.655 & 0.523 & 0.668 \\
\hline $\boldsymbol{K} \boldsymbol{L}\left[\boldsymbol{\zeta}_{\boldsymbol{j}}, \boldsymbol{\zeta}_{\boldsymbol{g} \mathbf{3}}\right]$ & 0.077 & 0.155 & 0.258 & 0.357 \\
\hline $\boldsymbol{K} \boldsymbol{L} \boldsymbol{C}\left[\boldsymbol{\zeta}_{\boldsymbol{j}}, \boldsymbol{\zeta}_{\boldsymbol{g} \mathbf{3}}\right]$ & 0.689 & 0.758 & 0.817 & 0.857 \\
\hline $\boldsymbol{K} \boldsymbol{L}\left[\boldsymbol{\zeta}_{\boldsymbol{j}}, \boldsymbol{\zeta}_{\boldsymbol{n}}\right]$ & 0.005 & 0.045 & 0.012 & 0.12 \\
\hline $\boldsymbol{K} \boldsymbol{L} \boldsymbol{C}\left[\boldsymbol{\zeta}_{\boldsymbol{j}}, \boldsymbol{\zeta}_{\boldsymbol{n}}\right]$ & 0.55 & 0.647 & 0.577 & 0.731 \\
\hline $\boldsymbol{K} \boldsymbol{L}\left[\boldsymbol{\zeta}_{\boldsymbol{g} \mathbf{1}}, \boldsymbol{\zeta}_{\boldsymbol{n}}\right]$ & 0.06 & 0.091 & 0.169 & 0.069 \\
\hline $\boldsymbol{K} \boldsymbol{L} \boldsymbol{C}\left[\boldsymbol{\zeta}_{\boldsymbol{g} \mathbf{1}}, \boldsymbol{\zeta}_{\boldsymbol{n}}\right]$ & 0.669 & 0.704 & 0.768 & 0.68 \\
\hline
\end{tabular}

Table 15: Posterior probability and identified models for the real-world time series.

\begin{tabular}{|c|c|c|c|c|c|c|c|c|c|c|}
\hline & \multicolumn{5}{|c|}{ Series A } & \multicolumn{5}{|c|}{ Series B } \\
\hline$p$ & $\zeta_{j}(p \mid y)$ & $\zeta_{g 1}(p \mid y)$ & $\zeta_{g 2}(p \mid y)$ & $\zeta_{g 3}(p \mid y)$ & $\zeta_{n}(p \mid y)$ & $\zeta_{j}(p \mid y)$ & $\zeta_{g 1}(p \mid y)$ & $\zeta_{g 2}(p \mid y)$ & $\zeta_{g 3}(p \mid y)$ & $\zeta_{n}(p \mid y)$ \\
\hline 1 & 0.811 & 0.929 & 0.853 & 0.925 & 0.821 & 0.811 & 0.881 & 0.789 & 0.865 & 0.719 \\
\hline 2 & 0.143 & 0.065 & 0.117 & 0.069 & 0.151 & 0.143 & 0.105 & 0.168 & 0.120 & 0.228 \\
\hline 3 & 0.032 & 0.005 & 0.02 & 0.005 & 0.021 & 0.032 & 0.010 & 0.029 & 0.011 & 0.043 \\
\hline 4 & 0.014 & 0.001 & 0.01 & 0.001 & 0.007 & 0.014 & 0.003 & 0.014 & 0.004 & 0.009 \\
\hline \multirow[t]{2}{*}{ Identified $p$} & $\mathrm{AR}(1)$ & $\mathrm{AR}(1)$ & $\operatorname{AR}(1)$ & $\operatorname{AR}(1)$ & $\operatorname{AR}(1)$ & $\operatorname{AR}(1)$ & $\operatorname{AR}(1)$ & $\operatorname{AR}(1)$ & $\operatorname{AR}(1)$ & $\operatorname{AR}(1)$ \\
\hline & \multicolumn{5}{|c|}{ Series C } & \multicolumn{5}{|c|}{ Series D } \\
\hline$p$ & $\zeta_{j}(p \mid y)$ & $\zeta_{g 1}(p \mid y)$ & $\zeta_{g 2}(p \mid y)$ & $\zeta_{g 3}(p \mid y)$ & $\zeta_{n}(p \mid y)$ & $\zeta_{j}(p \mid y)$ & $\zeta_{g 1}(p \mid y)$ & $\zeta_{g 2}(p \mid y)$ & $\zeta_{g 3}(p \mid y)$ & $\zeta_{n}(p \mid y)$ \\
\hline 1 & 0.000 & 0.001 & 0.000 & 0.001 & 0.000 & 0.067 & 0.268 & 0.127 & 0.27 & 0.146 \\
\hline 2 & 0.477 & 0.797 & 0.489 & 0.79 & 0.544 & 0.318 & 0.464 & 0.382 & 0.458 & 0.449 \\
\hline 3 & 0.18 & 0.114 & 0.163 & 0.116 & 0.181 & 0.443 & 0.24 & 0.409 & 0.244 & 0.341 \\
\hline 4 & 0.343 & 0.089 & 0.348 & 0.092 & 0.275 & 0.172 & 0.028 & 0.082 & 0.028 & 0.064 \\
\hline Identified $p$ & $\mathrm{AR}(2)$ & $\operatorname{AR}(2)$ & $\operatorname{AR}(2)$ & $\operatorname{AR}(2)$ & $\operatorname{AR}(2)$ & $\operatorname{AR}(3)$ & $\operatorname{AR}(2)$ & $\operatorname{AR}(3)$ & $\mathrm{AR}(2)$ & $\mathrm{AR}(2)$ \\
\hline
\end{tabular}




\section{Conclusions}

In this paper we first obtained the posterior mass functions of the AR model order by considering three types of priors for the AR model coefficients, namely Jeffreys', g, and natural conjugate priors, and three priors for the model order including uniform, arithmetic, and geometric priors. We then introduced the KL divergence and its calibration between the resulting posteriors to measure the distance between these posteriors. We used a large number of Monte Carlo simulations to evaluate the impact of the posteriors distance in the accuracy of model identification. Simulation results confirmed that the posteriors resulting from Jeffreys', g, and natural conjugate priors are strongly different, and the highest percentage of identified models is obtained from employing the g-prior distribution. Along with the simulation study, we applied our work to real-world time series datasets and their results are consistent with those of the simulation study and the non-Bayesian approach. Future work may be an extension to multivariate autoregressive models.

\section{References}

1. Abramowitz, M., and Stegun, I. A. (1972). Handbook of mathematical functions with formulas, graphs, and mathematical table. Dover Publications, Inc.

2. Berger, J. (1985). Statistical decision theory and Bayesian analysis. Springer.

3. Box, G. E. P., Jenkins, G. M., Reinsel, G. C., and Ljung, G. M. (2015). Time Series Analysis: Forecasting and Control. John Wiley \& Sons.

4. Broemeling, L. D. (1985). Bayesian analysis of linear models. CRC Press.

5. Contreras-Reyes, J. E., and Arellano-Valle R. B. (2012). Kullback-leibler divergence measure for multivariate skew-normal distributions. Entropy, 14(9): 1606-1626.

6. Diaz, J., and Farah, J. L. (1981). Bayesian identification of autoregressive process. In proceedings of the 22nd NBER-NSC Seminar on Bayesian Inference in Econo-metrics.

7. Fan, C., and Yao, S. (2009). Bayesian approach for arma process and its application. International Business Research, 1(4): 49-55.

8. Fernandez, C., Ley, E.. and Steel, M. F. (2001). Benchmark priors for bayesian model averaging. Journal of Econometrics, 100(2): 381-427.

9. Jeffreys, H. (1961). The theory of probability. Oxford University Press, London.

10. Kullback, S., and Leibler, R. A. (1951). On information and sufficiency. The annals of mathematical statistics, 22(1): 79-86.

11. McCulloch, R. E. (1989). Local model influence. Journal of the American Statistical Association, 84(406): 473-478.

12. Rachev, S. T., Hsu, J. S., Bagasheva, B. S., and Fabozzi, F. J. (2008). Bayesian methods in finance, volume 153. John Wiley \& Sons.

13. Schlaifer, R., and Raiffa, H. (1961). Applied statistical decision theory. Div. of Research, Graduate School of Business Administration, Harvard University. 
14. Shaarawy, S. M., and Ali, S. S. (2008). Bayesian identification of multivariate autoregressive processes. Communications in Statistics - Theory and Methods, 37(5): 791- 802.

15. Shaarawy, S. M., and Ali, S. S. (2012). Bayesian model order selection of vector mov $\neg$ ing average processes. Communications in Statistics - Theory and Methods, 41(4): 684-698.

16. Shaarawy, S. M., Soliman, E. E. A., and Ali, S. S. (2007). Bayesian identification of moving average models. Communications in Statistics - Theory and Methods, 36(12): 2301-2312.

17. Soliman, E. E. A., Shaarawy, S. M., and Sorour, W. W. (2015). On bayesian identification of autoregressive processes. Pakistan Journal of Statistics and Operation Research, 11(1): 2301-2312.

18. Zellner, A. (1986). On assessing prior distributions and bayesian regression analysis with g-prior distributions. Bayesian inference and decision techniques: Essays in Honor of Bruno De Finetti, 6: 233-243. 